Rasim M. Alguliyev ${ }^{1}$, Ramiz M. Aliguliyev ${ }^{2}$,

DOI: $10.25045 /$ jpit.v06.i2.03

Nigar T. Ismayilova ${ }^{3}$

Institute of Information Technology of ANAS, Baku, Azerbaijan

${ }^{1}$ rasim@ science.az, ${ }^{2}$ r.aliguliyev@gmail.com, ${ }^{3}$ nigar@iit.ab.az

\title{
IMPACT FACTOR WEIGHTED BY 5-YEAR IMPACT FACTOR
}

Impact factor is the most used indicator for journals ranking defined by citations frequency. Impact factor is the ratio of the number of citations in the current year to papers published in the previous two years to the number of substantive articles published within the same two years. In impact factor's calculation a number of all citations to journal, regardless of the prestige of cited journals, scientific units (paper, researcher, journal or scientific organization) is used, however, citations by journals with high impact factor or researchers with high Hirsch index are more important than citations by journals without impact factor or unknown researcher. In this paper we propose impact factor weighted by citing journals' 5-year impact factors for getting more accurate rankings for journals, which consider not only quantity of citations, but also quality of citing journals.

Keywords: impact factor; weighted impact factor; 5-year impact factor

\section{Introduction}

Today, a lot of scientific papers in different subject fields are published. So determining the influence of each paper or journal where these papers are published is very important. Nowadays the most used indicator for journal influence measuring is impact factor (IF) proposed by E. Garfield [1]. Generally accepted as an indicator of journal prestige the Thomson Reuters (former Institute of Scientific Information - ISI) calculates the IF of $j^{\text {th }}$ journal in year $t$ as ratio of number of citations in current year to articles published in this journal during previous two years to number of these articles:

$$
I F_{t}^{j}=\frac{\sum_{i=1}^{n_{j}^{t}} c_{i j}^{t}}{a_{j}^{t-1}+a_{j}^{t-2}},
$$

where $n_{j}^{t}$ - is the number of journals in the set of all journals indexed in year $t, c_{i j}^{t}-$ is the number of citations to journal $j$ from journal $i$ in year $t$ and $a_{j}^{t}-$ is the total number of articles published in year $t$. Although IF is an important indicator for evaluation of scientific journals used by librarians, researchers, science policymakers, there are many critiques against the IF efficiency [2-3]. Reedjik relates it to wrong, biased and even manipulated citations, as a result of citation habits for authors in different fields, selectivity in citations by authors, errors made by authors in references at the end of documents and by ISI in entering publications and citations in databases, in classifying citations and accrediting them to journals and authors. During the last decades different modifications of ISI IF [4] were proposed. For using with complex citation databases as Scopus in [5] the SCImago Journal Rank on citation weighting schemes and eigenvector centrality was proposed. Perez-Hornero et al. proposed a Bayesian approach to the problem with taking into account journals' recent trajectory besides the current prestige of a given journal [6]. Although during the last decades new indicators for journals evaluation were proposed, it was discovered that they (the Eigenvector, the SCImago journal rank, an Article Influence Score and journal h-index) correlate very much with the IF proposed by ISI and among each other [7]. One of the main disadvantages of IF is the equality of citations, regardless of the importance of the citing journals [8-10]. Unfortunately, in calculation of IF prestige, reputation of citing journals is not considered, nevertheless 10 citations from journal with high IF must be preferred to by one citation from 10 journals with low IF or without IF. For solution of this 
problem in this paper we propose an impact factor calculated by taking into account 5-year impact factor of each citing journal.

\section{Related works}

In general, journals in specialized and applied disciplines get more citations than journals in fundamental subject fields. The numbers of researchers and publishing journals in each field greatly affect the impact factor [11]. For this reason some researchers proposed methods for normalization of impact factors of journals for different scientific fields. Owlia et al. [12] introduced normalized impact factor for evaluating the quality of journals and research works in different disciplines. The normalized IF (NIF) index was established based on multiplication of journal IF by constant factor. NIF is the ratio of the aggregate impact factors of journals to aggregate impact factor of each discipline in given scientific field:

$$
N I F^{t}=\frac{C^{t} J^{t}}{A^{t} \hat{C}^{t}}
$$

where $C^{t}-$ is the total number of citations to the entire given scientific field in current year, $A^{t}$ - is the total number of articles published by journals from entire given scientific field in current year, $\hat{C}^{t}-$ is the total number of citations to the journals of the particular discipline and $J^{t}-$ is the total number of journals of the particular discipline published articles.

Another method for normalization of journal impact factors was produced by Iftikhar, Masood and Song [13]. Modified impact factors were calculated at disciplines, branches and specialities level and referred as Red, Yellow and Green MIF respectively. For modification the highest ISI IF of journal of that discipline, branch or specialty is weighted as 100 and other groups members are normalized accordingly by considering it as reference point. Also were proposed rank-normalized impact factor [14] and Source Normalized Impact per Paper (SNIP) by Scopus [15].

Weighting citations for more effective impact factor and more accurate ranking of journals have also been suggested by some researchers. Self-consistent methodology for determining citation influence weights for scientific journals, fields and subfields was developed in 1976. Also modification of IF using Weighted PageRank was proposed [16]. Authors suggested a simple combination of both the IF and the weighted PageRank and found that the resulting journal rankings well correspond to a general understanding of journal status [17]. Zyezkowski [18] formulated weighted impact factor of journals using weighted citations, weighted impact factor of papers, weighted Hirsch index and weighted efficiency index. In order not to ignore the impact or prestige of the cited journals different modifications of impact factor were proposed. Buela-Casal [19] had proposed two indices: a mean impact factor of the citing journals (MIFCJ) and a weighted impact factor (WIF). MIFCJ is ratio of multiplication of impact factors of citing journals by the number of cited articles to the total number citing articles published in previous two years:

$$
\operatorname{MIFJC}_{j}^{t}=\frac{\sum_{i=1}^{n_{j}^{t}} I F_{i}^{t-1} c_{i j}^{t}}{a_{j}^{t-1}+a_{j}^{t-2}} .
$$

Weighted impact factor of a journal is the average of MIFCJ and IF from ISI Journal Citation Ranking (JCR) reports:

$$
W I F_{j}^{t}=\frac{M I F J C_{j}^{t}+I F_{j}^{t-1}}{2}
$$

Habibzadeh and Yadollahie [20] suggested weighted impact factor with relative weights of journals by their IF in the previous year normalized using logistic function: 


$$
W I F_{i}^{j}=\frac{\sum_{j=1}^{n} w_{i j}^{t} \times c_{i j}^{t}}{a_{j}^{t-1}+a_{j}^{t-2}} .
$$

Here $w_{i j}^{t}$ denotes the weight of relative to $J_{i}$ weighted impact factor of journal $J_{k}$ in year $t$ :

$$
w_{i j}^{t}=10 \times \frac{1-0.828 \times e^{-q_{i j}^{t}}}{1+16.183 \times e^{-q_{i j}^{t}}}
$$

where $q_{i j}^{t}$ - is a ratio of the IF of citing journal in the previous year to the IF of cited journal in the previous year:

$$
q_{i j}^{t}=\frac{I F_{i}^{t-1}}{I F_{j}^{t-1}}
$$

Although proposed by researchers weighted IF (WIF) takes into account not only the number of times the journal has been cited, but also the prestige of the journals by which it has been cited. Some serious problems of proposed WIF were determined. Because of ranking of journals based in this WIF can be misleading. Waltman \& Van Eck [21] showed how the problems with the WIF proposed by Habibzadeh and Yadollahie (H\&Y) [20] can be solved.

In our opinion, the main drawback of WIF proposed by H\&Y is dependence on IF of cited journal in previous year.

Example: Let us calculate weighted impact factors of 3 journals for current year with the same number of articles in previous two years, we assume that number of citations and impact factors of citing journal to these articles in current year are the same for each journal (Table 1):

Number of articles: $P_{i}=20, i=1,2,3$.

Impact factors of journals in previous year: $I F_{1}=2 ; I F_{2}=4 ; I F_{3}=6$.

Table 1. Impact factors and numbers of citations of citing journals for journals in example.

\begin{tabular}{|l|c|c|}
\hline$N$ & IF of citing journal & Number of citations \\
\hline 1 & 2 & 2 \\
\hline 2 & 4 & 2 \\
\hline 3 & 8 & 2 \\
\hline 4 & 16 & 2 \\
\hline
\end{tabular}

Using given data were calculated weighted impact factors for given journals proposed by $\mathrm{H} \& \mathrm{Y}$ :

$$
W I F_{1}=0.994 ; W I F_{2}=0.760 ; W I F_{3}=0.444 .
$$

As result we can see that, calculated weighted impact factors for 3 journals with the same number of citations from the same sources are different depending on impact factors of journals in previous year. Cited journals with high impact factor in previous year gets lower weighted impact factors. This disadvantage of WIF by H\&Y makes ranking of journals by this method undesirable.

\section{Proposed weighted impact factor}

Assume that, $S^{t}$ - is the set of all journals indexed in year $t, n^{t}-$ is the number of indexed journals, $n_{j}^{t}$ - is the number of journals in $S^{t}$ cited the articles of journal $j, a_{j}^{t}$ - is the total number of articles published in year $t, c_{i j}{ }^{t}-$ is the number of citations to journal $j$ from journal $i$ in year $t, F I F_{j}^{t}$ - is 5-year impact factor of journal $j$ in year $t$. Proposed weighted IF of journal $j$ in year $t$ is 


$$
W I F_{j}^{t}=\frac{\sum_{i=1}^{n_{j}^{t}}\left(F I F_{i}^{t-1}+1\right) c_{i j}^{t}}{a_{j}^{t-1}+a_{j}^{t-2}} .
$$

To analyze the efficiency of proposed impact factor, we calculated new impact factors in 2013 for selected twenty journals in computer science fields from JCR for 2013 (Table 2).

Table 2

Some indicators of analyzed journal received from Scopus database

\begin{tabular}{|c|c|c|c|c|}
\hline $\mathrm{N}$ & Journal & $\begin{array}{c}\text { Number of } \\
\text { articles } \\
\text { published in } \\
2011 \text { and } \\
2012\end{array}$ & $\begin{array}{c}\text { Number of } \\
\text { citations in } 2013 \\
\text { to articles } \\
\text { published in } \\
2011 \text { and } 2012 \\
\end{array}$ & $\begin{array}{c}\text { Impact } \\
\text { factor of } \\
\text { journal in } \\
\text { previous } \\
\text { year }\end{array}$ \\
\hline 1. & Neural Computation & 226 & 383 & 1.76 \\
\hline 2. & Swarm Intelligence & 26 & 48 & 0.64 \\
\hline 3. & Neural Processing Letters & 76 & 94 & 1.24 \\
\hline 4. & Artificial Life & 48 & 93 & 1.585 \\
\hline 5. & Cognitive Computation & 88 & 97 & 0.867 \\
\hline 6. & Computer Speech And Language & 67 & 121 & 1.463 \\
\hline 7. & Fuzzy Optimization and Decision Making & 45 & 45 & 1.488 \\
\hline 8. & Genetic Programming and Evolvable Machines & 42 & 45 & 1.333 \\
\hline 9. & $\begin{array}{l}\text { International Journal of Appled Mathematics and } \\
\text { Computer Science }\end{array}$ & 136 & 189 & 1.008 \\
\hline 10. & Journal of Ambient Intelligence and Smart Environments & 74 & 80 & 1.298 \\
\hline 11. & ACM Transactions on Applied Perception & 40 & 42 & 1 \\
\hline 12. & ACM Transactions on Knowledge Discovery from Data & 37 & 42 & 1.676 \\
\hline 13. & Acm Transactions on Information Systems & 42 & 55 & 1.07 \\
\hline 14. & ACM Transactions on the Web & 39 & 62 & 1.405 \\
\hline 15. & ACM Transactions on Sensor Networks & 54 & 79 & 1.444 \\
\hline 16. & $\begin{array}{l}\text { Acm Transactions on Software Engineering And } \\
\text { Methodology }\end{array}$ & 37 & 54 & 1.548 \\
\hline 17. & $\begin{array}{l}\text { IEEE Transactions on Computational Intelligence and AI } \\
\text { in Games }\end{array}$ & 50 & 58 & 1.694 \\
\hline 18. & $\begin{array}{l}\text { IEEE Transactions on Dependable and Secure } \\
\text { Computing }\end{array}$ & 143 & 163 & 1.059 \\
\hline 19. & IEEE Transactions on Autonomous Mental Development & 54 & 73 & 2.17 \\
\hline 20. & World Wide Web & 58 & 94 & 1.196 \\
\hline
\end{tabular}

For these journals number of articles published in 2011 and 2012, total number of citations in 2013, number of sources citing journal were determined using data from Scopus database about articles and their citations. Also the numbers of citations from each journal with impact factors were determined for given journals (Table 3).

Table 3

5-year impact factors of citing journals and number of citations from them

\begin{tabular}{|c|c|c|c|c|c|c|c|c|}
\hline J1 - Neural Computation \\
\hline $\mathbf{1}$ & $\mathbf{2}$ & $\mathbf{3}$ & $\mathbf{4}$ & $\mathbf{5}$ & $\mathbf{6}$ & $\mathbf{7}$ & $\mathbf{8}$ & $\mathbf{9}$ \\
\hline $35.89(\mathbf{1})$ & $34.37(\mathbf{1})$ & $31.03(\mathbf{3})$ & $23.17(\mathbf{1})$ & $16.41(\mathbf{1})$ & $16.40(\mathbf{4})$ & $13.58(\mathbf{1})$ & $14.57(\mathbf{1})$ & $14.47(\mathbf{1})$ \\
\hline $\mathbf{1 0}$ & $\mathbf{1 1}$ & $\mathbf{1 2}$ & $\mathbf{1 3}$ & $\mathbf{1 4}$ & $\mathbf{1 5}$ & $\mathbf{1 6}$ & $\mathbf{1 7}$ & $\mathbf{1 8}$ \\
\hline $13.45(\mathbf{1})$ & $11.34(\mathbf{3})$ & $10.58(\mathbf{9})$ & $10.45(\mathbf{1})$ & $9.924(\mathbf{2})$ & $7.87(\mathbf{1 0})$ & $7.463(\mathbf{2})$ & $7.063(\mathbf{4})$ & $6.895(\mathbf{3})$ \\
\hline $\mathbf{1 9}$ & $\mathbf{2 0}$ & $\mathbf{2 1}$ & $\mathbf{2 2}$ & $\mathbf{2 3}$ & $\mathbf{2 4}$ & $\mathbf{2 5}$ & $\mathbf{2 6}$ & $\mathbf{2 7}$ \\
\hline $6.144(\mathbf{5})$ & $6(\mathbf{1})$ & $5.94(\mathbf{1 4})$ & $5.484(\mathbf{1})$ & $4.885(\mathbf{1})$ & $4.544(\mathbf{1})$ & $4.479(\mathbf{4})$ & $4.422(\mathbf{1})$ & $4.284(\mathbf{5})$ \\
\hline $\mathbf{2 8}$ & $\mathbf{2 9}$ & $\mathbf{3 0}$ & $\mathbf{3 1}$ & $\mathbf{3 2}$ & $\mathbf{3 3}$ & $\mathbf{3 4}$ & $\mathbf{3 5}$ & $\mathbf{3 6}$ \\
\hline
\end{tabular}


Problems of information technology, 2015, №2, 26-35

\begin{tabular}{|c|c|c|c|c|c|c|c|c|}
\hline $4.25(\mathbf{1})$ & 4.24 (11) & $4.049(7)$ & $4.017(\mathbf{1})$ & $3.879(2)$ & 3.844 (1) & $3.71(\mathbf{1})$ & 3.707 (1) & $3.676(\mathbf{1})$ \\
\hline 37 & 38 & 39 & 40 & 41 & 42 & 43 & 44 & 45 \\
\hline $3.668(2)$ & 3.646 (1) & $3.632(\mathbf{9})$ & $3.612(2)$ & $3.607(8)$ & $3.568(\mathbf{1})$ & 3.291 (1) & $3.219(\mathbf{1})$ & $3.146(\mathbf{1})$ \\
\hline 46 & 47 & 48 & 49 & 50 & 51 & 52 & 53 & 54 \\
\hline 3.108 (1) & $3.069(\mathbf{1})$ & $3.068(3)$ & $3.05(\mathbf{1})$ & $2.998(3)$ & $2.927(2)$ & $2.895(\mathbf{1})$ & $2.892(4)$ & $2.743(\mathbf{1})$ \\
\hline 55 & 56 & 57 & 58 & 59 & 60 & 61 & 62 & 63 \\
\hline $2.733(\mathbf{1})$ & $2.653(2)$ & $2.61(\mathbf{2 0})$ & $2.567(\mathbf{1})$ & $2.526(\mathbf{1})$ & $2.525(\mathbf{1})$ & $2.501(\mathbf{1 0})$ & $2.496(\mathbf{1})$ & $2.484(2)$ \\
\hline 64 & 65 & 66 & 67 & 68 & 69 & 70 & 71 & 72 \\
\hline $2.38(31)$ & $2.339(\mathbf{1})$ & $2.307(\mathbf{3})$ & $2.287(3)$ & $2.27(\mathbf{1})$ & $2.158(\mathbf{1})$ & $2.143(2)$ & $2(\mathbf{1})$ & $1.947(\mathbf{1})$ \\
\hline 73 & 74 & 75 & 76 & 77 & 78 & 79 & 80 & 81 \\
\hline $1.938(3)$ & $1.922(3)$ & $1.871(1)$ & $1.811(\mathbf{8})$ & $1.767(\mathbf{1})$ & $1.745(\mathbf{1})$ & $1.732(1)$ & 1.724 (1) & $1.643(\mathbf{1})$ \\
\hline 82 & 83 & 84 & 85 & 86 & 87 & 88 & 89 & 90 \\
\hline $1.6(\mathbf{1})$ & $1.596(\mathbf{1})$ & $1.595(\mathbf{1})$ & $1.572(\mathbf{1})$ & $1.55(\mathbf{1})$ & $1.529(2)$ & $1.402(2)$ & $1.386(2)$ & $1.338(\mathbf{1})$ \\
\hline 91 & 92 & 93 & 94 & 95 & 96 & 97 & 98 & 99 \\
\hline $1.336(\mathbf{1})$ & $1.319(\mathbf{1})$ & $1.314(\mathbf{1})$ & $1.305(\mathbf{1})$ & $1.231(\mathbf{1})$ & $1.216(\mathbf{1})$ & $1.192(\mathbf{1})$ & $1.183(\mathbf{1})$ & $1.182(\mathbf{1})$ \\
\hline 100 & 101 & 102 & 103 & 104 & 105 & 106 & 107 & 108 \\
\hline $1.14(3)$ & $1.074(\mathbf{1})$ & $1.032(\mathbf{1})$ & $0.97(\mathbf{1})$ & 0.84 (1) & 0.816 (1) & 0.697 (1) & $0.672(2)$ & $0.6(\mathbf{1})$ \\
\hline 109 & 110 & 111 & & & & & & \\
\hline 0.548 (1) & 0.297 (1) & 0.187 (2) & & & & & & \\
\hline \multicolumn{9}{|c|}{ J2-Swarm Intelligence } \\
\hline 1 & 2 & 3 & 4 & 5 & 6 & 7 & 8 & 9 \\
\hline $6.226(1)$ & $5.165(2)$ & $3.448(\mathbf{1})$ & $3.405(\mathbf{1})$ & $3.097(2)$ & $2.747(\mathbf{1})$ & $1.957(\mathbf{1})$ & $1.615(\mathbf{1})$ & $1.545(2)$ \\
\hline 10 & 11 & & & & & & & \\
\hline $1.364(\mathbf{1})$ & $0.953(2)$ & & & & & & & \\
\hline \multicolumn{9}{|c|}{ J3-Neural Processing Letters } \\
\hline 1 & 2 & 3 & 4 & 5 & 6 & 7 & 8 & 9 \\
\hline $7.854(\mathbf{1})$ & $4.268(\mathbf{1})$ & $3.676(1)$ & $3.632(2)$ & $3.513(\mathbf{1})$ & $3.219(\mathbf{1})$ & $2.501(2)$ & $2.457(3)$ & $2.384(2)$ \\
\hline 10 & 11 & 12 & 13 & 14 & 15 & 16 & 17 & 18 \\
\hline $2.339(\mathbf{1})$ & $2.143(\mathbf{1})$ & $1.831(\mathbf{1})$ & $1.811(8)$ & $1.71(\mathbf{1})$ & $1.529(\mathbf{1})$ & $1.454(2)$ & $1.42(\mathbf{1})$ & $1.36(\mathbf{1})$ \\
\hline 19 & 20 & 21 & 22 & 23 & 24 & 25 & 26 & 27 \\
\hline $1.329(\mathbf{1})$ & $1.23(\mathbf{1 0})$ & $1.216(1)$ & $1.183(2)$ & $1.074(6)$ & $1.04(3)$ & $0.898(\mathbf{1})$ & $0.866(\mathbf{1})$ & $0.84(2)$ \\
\hline 28 & 29 & 30 & 31 & 32 & 33 & 34 & 35 & 36 \\
\hline $0.774(5)$ & $0.755(\mathbf{1})$ & $0.753(\mathbf{1})$ & $0.716(\mathbf{1})$ & $0.682(5)$ & $0.64(1)$ & $0.622(\mathbf{1})$ & 0.561 (1) & $0.497(2)$ \\
\hline \multicolumn{9}{|c|}{ J4-Artificial Life } \\
\hline 1 & 2 & 3 & 4 & 5 & 6 & 7 & 8 & 9 \\
\hline $17.72(\mathbf{1})$ & $13.56(\mathbf{1})$ & 7.51 (1) & 7.435 (1) & 6.69 (1) & $6.226(\mathbf{1})$ & $5.165(2)$ & $4.728(\mathbf{1})$ & $4.446(\mathbf{1})$ \\
\hline 10 & 11 & 12 & 13 & 14 & 15 & 16 & 17 & 18 \\
\hline $4.406(\mathbf{1})$ & $4.244(7)$ & 2.496 (1) & $2.333(\mathbf{1})$ & $2.307(\mathbf{1})$ & $2(\mathbf{1})$ & 1.945 (1) & $1.777(\mathbf{1})$ & $1.545(2)$ \\
\hline 19 & 20 & 21 & 22 & 23 & 24 & 25 & 26 & \\
\hline $1.454(\mathbf{1})$ & 1.364 (1) & $1.336(\mathbf{1})$ & $0.953(2)$ & 0.816 (1) & 0.617 (1) & 0.48 (1) & 0.417 (1) & \\
\hline \multicolumn{9}{|c|}{ J5-Cognitive Computation } \\
\hline 1 & 2 & 3 & 4 & 5 & 6 & 7 & 8 & 9 \\
\hline $9.924(\mathbf{1})$ & 7.869 (1) & $7.298(\mathbf{1})$ & 4.479 (1) & $4.372(\mathbf{1})$ & $4.244(3)$ & 4.017 (1) & $3.674(\mathbf{1})$ & 3.598 (1) \\
\hline 10 & 11 & 12 & 13 & 14 & 15 & 16 & 17 & 18 \\
\hline $3.262(\mathbf{1})$ & $2.998(2)$ & $2.847(\mathbf{1})$ & 2.538 (1) & 2.525 (1) & $2.501(\mathbf{3})$ & $2.445(\mathbf{1})$ & $2.339(\mathbf{1})$ & $2.194(\mathbf{1})$ \\
\hline 19 & 20 & 21 & 22 & 23 & 24 & 25 & 26 & 27 \\
\hline $2.158(\mathbf{1})$ & $1.938(2)$ & $1.936(\mathbf{1})$ & $1.811(2)$ & 1.745 (1) & $1.596(\mathbf{1})$ & $1.529(2)$ & $1.52(\mathbf{1})$ & $1.423(\mathbf{1})$ \\
\hline 28 & 29 & 30 & 31 & 32 & 33 & & & \\
\hline $1.157(2)$ & $1.14(14)$ & $0.846(\mathbf{1})$ & $0.735(\mathbf{1})$ & $0.592(\mathbf{1})$ & $0.326(\mathbf{1})$ & & & \\
\hline \multicolumn{9}{|c|}{ J6-Computer Speech And Language } \\
\hline 1 & 2 & 3 & 4 & 5 & 6 & 7 & 8 & 9 \\
\hline $7.694(2)$ & $2.643(1)$ & $2.395(2)$ & $2.339(2)$ & $1.952(2)$ & $1.936(\mathbf{1})$ & $1.915(2)$ & $1.708(\mathbf{1})$ & $1.52(8)$ \\
\hline 10 & 11 & 12 & 13 & 14 & 15 & 16 & 17 & 18 \\
\hline $1.423(5)$ & $1.41(\mathbf{1})$ & $1.146(\mathbf{1})$ & $1.137(4)$ & $1.074(2)$ & $0.977(\mathbf{1})$ & $0.959(\mathbf{1})$ & $0.932(\mathbf{1})$ & $0.767(\mathbf{1})$ \\
\hline 19 & 20 & 21 & 22 & 23 & & & & \\
\hline 0.664 (1) & $0.617(\mathbf{1})$ & $0.505(1)$ & $0.466(1)$ & 0.305 (1) & & & & \\
\hline \multicolumn{9}{|c|}{ J7 - Fuzzy Optimization and Decision Making } \\
\hline 1 & 2 & 3 & 4 & 5 & 6 & 7 & 8 & 9 \\
\hline
\end{tabular}




\begin{tabular}{|c|c|c|c|c|c|c|c|c|}
\hline $3.676(3)$ & $2.218(2)$ & $2.167(\mathbf{1})$ & $2.165(1)$ & $1.81(\mathbf{1 5})$ & $1.721(\mathbf{1})$ & $1.674(2)$ & $1.579(2)$ & $1.386(\mathbf{1})$ \\
\hline 10 & 11 & 12 & 13 & 14 & 15 & & & \\
\hline $1.364(4)$ & $1.183(2)$ & $0.846(2)$ & $0.746(1)$ & $0.612(\mathbf{1})$ & $0.27(16)$ & & & \\
\hline \multicolumn{9}{|c|}{ J8-Genetic Programming and Evolvable Machines } \\
\hline 1 & 2 & 3 & 4 & 5 & 6 & 7 & 8 & 9 \\
\hline $3.676(\mathbf{1})$ & $3.027(\mathbf{1})$ & $2.526(1)$ & $2.501(\mathbf{1})$ & $1.938(\mathbf{1})$ & $1.811(\mathbf{1})$ & $1.795(\mathbf{1})$ & $1.726(2)$ & $1.625(\mathbf{1})$ \\
\hline 10 & 11 & 12 & 13 & & & & & \\
\hline $1.39(\mathbf{1})$ & $1.349(\mathbf{1})$ & $1.282(8)$ & $1.231(\mathbf{1})$ & & & & & \\
\hline \multicolumn{9}{|c|}{ J9-International Journal of Appled Mathematics and Computer Science } \\
\hline $\mathbf{1}$ & 2 & 3 & 4 & 5 & 6 & 7 & 8 & 9 \\
\hline $4.244(1)$ & $3.676(1)$ & $3.601(1)$ & $3.212(1)$ & $2.64(2)$ & $2.62(1)$ & $2.457(1)$ & $2.382(1)$ & $2.255(1)$ \\
\hline 10 & 11 & 12 & 13 & 14 & 15 & 16 & 17 & 18 \\
\hline $2.151(1)$ & $2.04(4)$ & $1.758(1)$ & $1.674(1)$ & $1.651(1)$ & $1.625(1)$ & $1.529(2)$ & $1.504(1)$ & $1.454(1)$ \\
\hline 19 & 20 & 21 & 22 & 23 & 24 & 25 & 26 & 27 \\
\hline $1.368(2)$ & $1.364(2)$ & $1.359(1)$ & $1.289(2)$ & $1.216(3)$ & $1.201(2)$ & $1.183(2)$ & $1.182(1)$ & $1.158(1)$ \\
\hline 28 & 29 & 30 & 31 & 32 & 33 & 34 & 35 & 36 \\
\hline $1.15(52)$ & $1.024(1)$ & $0.932(1)$ & $0.898(2)$ & $0.829(1)$ & $0.8(4)$ & 0.671 (1) & $0.61(1)$ & $0.594(3)$ \\
\hline 37 & 38 & 39 & 40 & 41 & 42 & 43 & & \\
\hline $0.548(1)$ & $0.483(1)$ & $0.436(1)$ & $0.41(1)$ & $0.395(1)$ & $0.37(1)$ & $0.269(1)$ & & \\
\hline \multicolumn{9}{|c|}{ J10 - Journal of Ambient Intelligence and Smart Environments } \\
\hline 1 & 2 & 3 & 4 & 5 & 6 & 7 & 8 & 9 \\
\hline $3.382(\mathbf{1})$ & $2.7(\mathbf{1})$ & $2.632(1)$ & $2.525(1)$ & $2.339(\mathbf{1})$ & $2.003(\mathbf{1})$ & $1.947(\mathbf{1})$ & $1.811(2)$ & $1.64(16)$ \\
\hline 10 & 11 & & & & & & & \\
\hline $1.529(1)$ & $1.169(\mathbf{1})$ & & & & & & & \\
\hline \multicolumn{9}{|c|}{ J11-ACM Transactions on Applied Perception } \\
\hline 1 & 2 & 3 & 4 & 5 & 6 & 7 & 8 & 9 \\
\hline $6.144(1)$ & $4.283(2)$ & $4.017(\mathbf{1})$ & $2.62(1)$ & $2.61(1)$ & $2.566(1)$ & $2.445(2)$ & $2.395(\mathbf{1})$ & $2.292(\mathbf{1})$ \\
\hline 10 & 11 & 12 & 13 & 14 & 15 & 16 & 17 & 18 \\
\hline $2.007(\mathbf{1})$ & $2(\mathbf{1})$ & $1.905(\mathbf{1})$ & $1.36(2)$ & $1.269(5)$ & $1.216(\mathbf{1})$ & $1.112(\mathbf{1})$ & 0.675 (1) & $0.5(\mathbf{1})$ \\
\hline \multirow{2}{*}{\multicolumn{9}{|c|}{$\begin{array}{c}\mathbf{1 9} \\
0.453(\mathbf{1})\end{array}$}} \\
\hline & & & & & & & & \\
\hline \multicolumn{9}{|c|}{ J12 - ACM Transactions on Knowledge Discovery from Data } \\
\hline 1 & 2 & 3 & 4 & 5 & 6 & 7 & 8 & 9 \\
\hline $4.395(\mathbf{1})$ & $4.244(\mathbf{1})$ & $4.017(\mathbf{1})$ & $3.959(\mathbf{1})$ & $3.676(3)$ & $3.371(\mathbf{1})$ & $3.263(\mathbf{1})$ & $3.068(\mathbf{1})$ & $2.426(1)$ \\
\hline 10 & 11 & 12 & 13 & 14 & 15 & & & \\
\hline $2.339(3)$ & $1.838(\mathbf{1})$ & $1.811(\mathbf{1})$ & $1.359(2)$ & $0.739(\mathbf{1})$ & $0.707(\mathbf{1})$ & & & \\
\hline \multicolumn{9}{|c|}{ J13 - ACM Transactions on Information Systems } \\
\hline 1 & 2 & 3 & 4 & 5 & 6 & 7 & $\mathbf{8}$ & 9 \\
\hline $3.676(\mathbf{1})$ & $3.371(3)$ & $3.037(2)$ & $2.566(1)$ & $2.446(\mathbf{1})$ & $2.339(2)$ & $1.745(\mathbf{1})$ & $1.716(3)$ & $1.586(\mathbf{1})$ \\
\hline 10 & 11 & 12 & & & & & & \\
\hline $1.318(\mathbf{1})$ & $1.109(2)$ & $0.466(1)$ & & & & & & \\
\hline \multicolumn{9}{|c|}{ J14-ACM Transactions on the Web } \\
\hline 1 & 2 & 3 & 4 & 5 & 6 & 7 & 8 & 9 \\
\hline $7.854(2)$ & $4.395(\mathbf{1})$ & $3.676(2)$ & $3.371(\mathbf{1})$ & $3.037(2)$ & $2.927(\mathbf{1})$ & $2.446(5)$ & $2.424(2)$ & $2.339(3)$ \\
\hline 10 & 11 & 12 & 13 & 14 & 15 & 16 & 17 & 18 \\
\hline $2.158(3)$ & $2.033(\mathbf{1})$ & $1.469(1)$ & $1.452(\mathbf{1})$ & $1.388(2)$ & $1.384(\mathbf{1})$ & $1.322(2)$ & $1.109(\mathbf{1})$ & $0.943(2)$ \\
\hline 19 & 20 & 21 & & & & & & \\
\hline $0.785(\mathbf{1})$ & $0.765(\mathbf{1})$ & $0.605(\mathbf{1})$ & & & & & & \\
\hline \multicolumn{9}{|c|}{ J15-ACM Transactions on Sensor Networks } \\
\hline 1 & 2 & 3 & 4 & 5 & 6 & 7 & 8 & 9 \\
\hline $6.348(2)$ & $6.146(1)$ & $3.587(2)$ & $3.371(\mathbf{1})$ & $2.747(\mathbf{1})$ & $2.485(1)$ & $2.395(\mathbf{1})$ & $2.203(\mathbf{1})$ & $1.957(\mathbf{1})$ \\
\hline 10 & 11 & 12 & 13 & 14 & 15 & 16 & 17 & 18 \\
\hline $1.859(\mathbf{1})$ & $1.758(2)$ & $1.227(\mathbf{1})$ & $1.183(4)$ & $1.169(1)$ & $1.092(1)$ & $1.002(\mathbf{1})$ & $0.765(1)$ & $0.605(1)$ \\
\hline \multicolumn{9}{|l|}{19} \\
\hline \multicolumn{9}{|l|}{$0.43(\mathbf{5})$} \\
\hline \multicolumn{9}{|c|}{ J16-ACM Transactions on Software Engineering And Methodology } \\
\hline $\mathbf{1}$ & 2 & 3 & 4 & 5 & 6 & 7 & 8 & 9 \\
\hline $3.612(\mathbf{1})$ & $3.371(\mathbf{1})$ & $2.063(3)$ & $2.031(2)$ & $1.756(\mathbf{1})$ & $1.692(2)$ & $1.322(\mathbf{1})$ & $1.167(4)$ & $1.867(\mathbf{1})$ \\
\hline
\end{tabular}


Problems of information technology, 2015, №2, 26-35

\begin{tabular}{|c|c|c|c|c|c|c|c|c|}
\hline 10 & 11 & 12 & 13 & 14 & 15 & 16 & 17 & 18 \\
\hline $0.819(\mathbf{1})$ & $0.785(\mathbf{1})$ & $0.727(\mathbf{1})$ & $0.721(\mathbf{1})$ & $0.682(2)$ & $0.43(\mathbf{1})$ & $0.336(2)$ & $0.269(1)$ & $0.268(\mathbf{1})$ \\
\hline \multicolumn{9}{|c|}{ J17 - IEEE Transactions on Computational Intelligence and AI in Games } \\
\hline 1 & 2 & 3 & 4 & 5 & 6 & 7 & 8 & 9 \\
\hline $3.212(\mathbf{1})$ & $3.071(\mathbf{1})$ & $2.339(2)$ & $1.936(\mathbf{1})$ & $1.63(11)$ & $1.282(\mathbf{1})$ & $0.954(\mathbf{1})$ & $0.832(\mathbf{1})$ & $0.723(3)$ \\
\hline \multicolumn{9}{|c|}{ J18 - IEEE Transactions on Dependable and Secure Computing } \\
\hline 1 & 2 & 3 & 4 & 5 & 6 & 7 & 8 & 9 \\
\hline $6.895(\mathbf{1})$ & $3.676(4)$ & $3.371(2)$ & $3.191(\mathbf{1})$ & $3.071(3)$ & $2.744(\mathbf{1})$ & $2.426(\mathbf{1})$ & $2.339(2)$ & $2.259(4)$ \\
\hline 10 & 11 & 12 & 13 & 14 & 15 & 16 & 17 & 18 \\
\hline $2.067(\mathbf{1})$ & $2.021(\mathbf{1})$ & $1.894(\mathbf{1})$ & $1.726(\mathbf{1 0})$ & $1.576(2)$ & 1.42 (1) & $1.39(\mathbf{1})$ & $1.341(\mathbf{1})$ & $1.322(2)$ \\
\hline 19 & 20 & 21 & 22 & 23 & 24 & 25 & 26 & 27 \\
\hline $1.317(\mathbf{1})$ & $1.291(\mathbf{1})$ & $1.234(\mathbf{1})$ & $1.227(\mathbf{1})$ & $1.106(\mathbf{1})$ & $1.093(3)$ & $1.019(\mathbf{1})$ & $0.954(2)$ & 0.945 (1) \\
\hline 28 & 29 & 30 & 31 & 32 & 33 & 34 & 35 & 36 \\
\hline 0.874 (1) & $0.867(2)$ & 0.819 (1) & 0.793 (1) & $0.712(\mathbf{1})$ & 0.701 (1) & $0.697(3)$ & $0.625(2)$ & 0.606 (1) \\
\hline 37 & 38 & 39 & 40 & 41 & 42 & 43 & & \\
\hline 0.605 (1) & $0.497(2)$ & $0.463(2)$ & 0.43 (3) & $0.395(2)$ & 0.297 (1) & 0.187 (1) & & \\
\hline \multicolumn{9}{|c|}{ J19 - IEEE Transactions on Autonomous Mental Development } \\
\hline 1 & 2 & 3 & 4 & 5 & 6 & 7 & 8 & 9 \\
\hline $4.244(4)$ & $3.587(1)$ & $2.501(4)$ & $2.26(17)$ & $2.202(\mathbf{1})$ & $1.947(\mathbf{1})$ & $1.859(\mathbf{1})$ & $1.615(3)$ & $1.545(2)$ \\
\hline 10 & 11 & 12 & 13 & 14 & 15 & 16 & 17 & \\
\hline $1.529(1)$ & $1.423(1)$ & $1.137(\mathbf{1})$ & $1.043(\mathbf{1})$ & $0.898(\mathbf{1})$ & $0.817(\mathbf{1})$ & $0.675(\mathbf{1})$ & $0.567(1)$ & \\
\hline \multicolumn{9}{|c|}{ J20 - World Wide Web } \\
\hline 1 & 2 & 3 & 4 & 5 & 6 & 7 & 8 & 9 \\
\hline $3.676(1)$ & $3.219(2)$ & $3.191(\mathbf{1})$ & $2.446(1)$ & $2.426(1)$ & $2.403(1)$ & $2.339(2)$ & $2.031(\mathbf{1})$ & $1.955(\mathbf{1})$ \\
\hline 10 & 11 & 12 & 13 & 14 & 15 & 16 & 17 & 18 \\
\hline $1.838(\mathbf{1})$ & $1.586(\mathbf{1})$ & $1.45(23)$ & $1.251(\mathbf{1})$ & $1.169(\mathbf{1})$ & 0.94 (1) & $0.332(\mathbf{1})$ & $0.302(\mathbf{1})$ & 0.174 (1) \\
\hline
\end{tabular}

Note: here $(*)$ denotes the number of citations

Weighted impact factors of given journals were compared with JCR IF for 2013, WIF proposed by H\&Y, G. Buela-Casal and impact factors weighted by average and median of citing journals' impact factors and 5-year impact factors (Table 4).

Table 4

JCR IF, WIF by H\&Y, by Buela-Casal, proposed WIF, average and median WIF, weighted by average and median of 5-year impact factors for analyzed journals

\begin{tabular}{|l|l|c|c|c|c|c|c|c|c|}
\hline$N$ & \multicolumn{1}{|c|}{ Journal } & JCR IF & $\begin{array}{c}\text { WIF by } \\
\text { H\&Y }\end{array}$ & $\begin{array}{c}\text { WIF by G. } \\
\text { Buela- } \\
\text { Casal }\end{array}$ & $\begin{array}{c}\text { Proposed } \\
\text { WIF }\end{array}$ & $\begin{array}{c}\text { Average } \\
\text { WIF }\end{array}$ & $\begin{array}{c}\text { Median } \\
\text { WIF }\end{array}$ & $\begin{array}{c}\text { Average } \\
5 W I F\end{array}$ & $\begin{array}{c}\text { Median } \\
5 W I F\end{array}$ \\
\hline 1. & $\begin{array}{l}\text { Neural } \\
\text { Computation }\end{array}$ & $1.694[4]$ & $4.200[2]$ & $3.603[1]$ & $7.544[1]$ & $6.533[1]$ & $4.200[2]$ & $7.725[1]$ & $4.9[3]$ \\
\hline 2. & Swarm Intelligence & $1.833[2]$ & $4.457[1]$ & $1.577[9]$ & $3.472[4]$ & $3.238[3]$ & $2.055[8]$ & $5.204[3]$ & $5.071[2]$ \\
\hline 3. & $\begin{array}{l}\text { Neural Processing } \\
\text { Letters }\end{array}$ & $1.234[12]$ & $1.631[12]$ & $1.455[13]$ & $2.763[8]$ & $1.765[14]$ & $1.534[15]$ & $2.02[13]$ & $1.523[17]$ \\
\hline 4. & Artificial Life & $1.93[1]$ & $3.028[3]$ & $2.688[2]$ & $4.762[2]$ & $6.474[2]$ & $4.518[1]$ & $7.724[2]$ & $8.223[1]$ \\
\hline 5. & $\begin{array}{l}\text { Cognitive } \\
\text { Computation }\end{array}$ & $1.1[16]$ & $2.649[4]$ & $1.317[17]$ & $2.594[11]$ & $2.253[9]$ & $1.955[10]$ & $2.631[11]$ & $1.996[12]$ \\
\hline 6. & $\begin{array}{l}\text { Computer Speech } \\
\text { And Language }\end{array}$ & $1.812[3]$ & $1.915[8]$ & $1.778[7]$ & $2.875[6]$ & $2.137[11]$ & $2.317[4]$ & $3.091[6]$ & $2.57[8]$ \\
\hline 7. & $\begin{array}{l}\text { Fuzzy } \\
\text { Optimization and } \\
\text { Decision Making }\end{array}$ & $1[20]$ & $0.855[20]$ & $1.330[16]$ & $2.158[15]$ & $1.172[20]$ & $1.124[20]$ & $1.33[20]$ & $1.48[20]$ \\
\hline 8. & $\begin{array}{l}\text { Genetic } \\
\text { Programming and } \\
\text { Evolvable } \\
\text { Machines }\end{array}$ & $1.07[18]$ & $1.193[18]$ & $1.363[15]$ & $1.919[18]$ & $1.729[15]$ & $1.453[16]$ & $1.88[17]$ & $1.508[18]$ \\
\hline 9. & $\begin{array}{l}\text { International } \\
\text { Journal of Applied } \\
\text { Mathematics and } \\
\text { Computer Science }\end{array}$ & $1.39[9]$ & $1.698[11]$ & $1.266[19]$ & $2.443[12]$ & $1.643[18]$ & $1.401[18]$ & $1.815[18]$ & $1.593[16]$ \\
\hline
\end{tabular}


Problems of information technology, 2015, №2, 26-35

\begin{tabular}{|c|c|c|c|c|c|c|c|c|c|}
\hline 10. & $\begin{array}{l}\text { Journal of Ambient } \\
\text { Intelligence and } \\
\text { Smart } \\
\text { Environments }\end{array}$ & 1.08 [17] & 1.198 [17] & 1.294 [18] & $1.758[20]$ & $1.658[17]$ & 1.403 [17] & 2.006 [14] & $1.773[14]$ \\
\hline 11. & $\begin{array}{l}\text { ACM Transactions } \\
\text { on Applied } \\
\text { Perception } \\
\end{array}$ & 1.05 [19] & $2.021[7]$ & 1.264 [20] & $2.426[13]$ & $1.821[12]$ & 1.700 [12] & 2.223 [12] & $2.05[11]$ \\
\hline 12 . & $\begin{array}{l}\text { ACM Transactions } \\
\text { on Knowledge } \\
\text { Discovery from } \\
\text { Data }\end{array}$ & $1.145[14]$ & 1.573 [13] & $1.806[6]$ & 2.61 [9] & $2.480[6]$ & 2.105 [7] & 3.099 [5] & $3.118[5]$ \\
\hline 13. & $\begin{array}{l}\text { Acm Transactions } \\
\text { On Information } \\
\text { Systems }\end{array}$ & $1.3[11]$ & $2.059[6]$ & $1.389[14]$ & $2.3[14]$ & $2.406[7]$ & $2.132[6]$ & 2.897 [9] & $3.063[6]$ \\
\hline 14. & $\begin{array}{l}\text { ACM Transactions } \\
\text { on the Web }\end{array}$ & $1.595[6]$ & $2.076[5]$ & $1.829[5]$ & 3.828 [3] & $2.670[4]$ & $2.234[5]$ & 3.855 [4] & 3.718 [4] \\
\hline 15 . & $\begin{array}{l}\text { ACM Transactions } \\
\text { on Sensor } \\
\text { Networks } \\
\end{array}$ & $1.463[8]$ & $1.697[10]$ & $1.615[8]$ & $2.607[10]$ & $2.285[8]$ & 1.612 [14] & 3.013 [7] & $2.184[10]$ \\
\hline 16. & $\begin{array}{l}\text { ACM Transactions } \\
\text { On Software } \\
\text { Engineering And } \\
\text { Methodology }\end{array}$ & 1.472 [7] & 1.369 [16] & $1.487[12]$ & $2.098[16]$ & $1.674[16]$ & 1.660 [13] & 1.912 [15] & $1.707[15]$ \\
\hline 17. & $\begin{array}{l}\text { IEEE Transactions } \\
\text { on Computational } \\
\text { Intelligence and AI } \\
\text { in Games }\end{array}$ & $\begin{array}{l}1.167 \\
{[13]}\end{array}$ & 1.139 [19] & 1.549 [10] & 1.88 [19] & $1.804[13]$ & 1.965 [9] & $1.899[16]$ & $1.885[13]$ \\
\hline 18. & $\begin{array}{l}\text { IEEE Transactions } \\
\text { on Dependable and } \\
\text { Secure Computing }\end{array}$ & $\begin{array}{l}1.137 \\
{[15]}\end{array}$ & $1.533[14]$ & $1.195[4]$ & 1.985 [17] & 1.534 [19] & 1.230 [19] & 1.812 [19] & 1.507 [19] \\
\hline 19. & $\begin{array}{l}\text { IEEE Transactions } \\
\text { on Autonomous } \\
\text { Mental } \\
\text { Development }\end{array}$ & $\begin{array}{l}1.348 \\
{[10]}\end{array}$ & $1.450[15]$ & $19[3]$ & $3.222[5]$ & ] & [3] & $2.929[8]$ & $3.053[7]$ \\
\hline 20. & World Wide Web & $1.623[5]$ & 1.862 [9] & $1.547[11]$ & $2.832[7]$ & $2.200[10]$ & $1.938[11]$ & $2.71[10]$ & 2.353 [9] \\
\hline
\end{tabular}

\section{Experiments and Discussions}

For describing the efficiency of proposed method Pearson correlation coefficients were calculated between sets of indictors (JCR IF, WIF by H \& Y, WIF by Buela-Casal, proposed WIF, average and median WIF, average and median 5WIF) (Table 5). Analyzing correlation coefficients among different indicators for journal evaluation, we can notice that, proposed in this paper WIF have high correlations with all analyzed indicators, excluding WIF proposed by Buela-Casal [19], which has low correlations with all indicators in this list. The WIF proposed by H\&Y [20] and WIF proposed by Buela-Casal [19] have the worst correlations, because these two indicators deny each other: WIF proposed by H\&Y decreases by increasing of cited journal IF in previous year, but WIF proposed by Buela-Casal [19] increases in the same case. The highest correlations are observed between average and median 5-year weighted impact factors.

Table 5

Pairwise Pearson correlations between impact factors

\begin{tabular}{|l|c|c|c|c|c|c|c|c|}
\hline Indicators & JCR IF & $\begin{array}{c}\text { Proposed } \\
\text { WIF }\end{array}$ & $\begin{array}{c}\text { WIF by } H \\
\& Y\end{array}$ & $\begin{array}{c}\text { WIF by G. } \\
\text { Buela-Casal }\end{array}$ & $\begin{array}{c}\text { Average } \\
\text { WIF }\end{array}$ & $\begin{array}{c}\text { Median } \\
\text { WIF }\end{array}$ & $\begin{array}{c}\text { Average } \\
5 \text { WIF }\end{array}$ & $\begin{array}{c}\text { Median } \\
5 \text { WIF }\end{array}$ \\
\hline JCR IF & 1 & & & & & & & \\
\hline $\begin{array}{l}\text { Proposed } \\
\text { WIF }\end{array}$ & 0.7398 & 1 & & & & & & \\
\hline $\begin{array}{l}\text { WIF by } H \\
\& Y\end{array}$ & 0.6120 & 0.7384 & 1 & & & & & \\
\hline WIF by G. & 0.6105 & 0.6226 & 0.2797 & 1 & & & & \\
\hline
\end{tabular}




\begin{tabular}{|l|c|c|c|c|c|c|c|c|}
\hline $\begin{array}{l}\text { Buela- } \\
\text { Casal }\end{array}$ & & & & & & & & \\
\hline $\begin{array}{l}\text { Average } \\
\text { WIF }\end{array}$ & 0.6256 & 0.8060 & 0.7414 & 0.6210 & 1 & & & \\
\hline $\begin{array}{l}\text { Median } \\
\text { WIF }\end{array}$ & 0.6361 & 0.7233 & 0.6045 & 0.6421 & 0.8887 & 1 & & \\
\hline $\begin{array}{l}\text { Average } \\
\text { WWIF }\end{array}$ & 0.7218 & 0.8135 & 0.8075 & 0.5774 & 0.9428 & 0.8647 & 1 & \\
\hline $\begin{array}{l}\text { Median } \\
\text { 5WIF }\end{array}$ & 0.6932 & 0.7699 & 0.7473 & 0.5790 & 0.9489 & 0.8948 & 0.9474 & 1 \\
\hline
\end{tabular}

\section{Conclusions}

As the special case from equation of proposed weighted impact factor we get current equation of impact factor calculated by ISI for JCR, but considering prestige and influence of cited journals gives more adequate indicator for journal ranking. Proposed method for IF can be also applied for researchers, organizations or country evaluation. Obviously, the paper or researcher cited by e.g. L. Zadeh is more important than paper cited by unknown researcher. Therefore, considering indexes of cited objects for research evaluation contributes more effective results.

\section{References}

1. Garfield E. Science citation index - a new dimension in indexing // Science, 1964, vol.144, no.3619, pp.649-654.

2. Seglen P.O. Citations and journal impact factors: questionable indicators of research quality // Allergy: European Journal of Allergy and Clinical Immunology, 1997, vol.52, no.11, pp.1050-1056.

3. Opthof T. Sense and nonsense about the impact factor // Cardiovascular Research, 1997, vol.33, no.1, pp.1-7.

4. Reedjik J. Sense and nonsense of science citation analyses: comments on the monopoly position of ISI and citation inaccuracies. Risks of possible misuse and biased citation and impact data // New Journal of Chemistry, 1998, vol.22, no.8, pp.767-770.

5. Gonzales-Pereira B, Guerrero-Bote V.P., Moya-Anegon F. A new approach to the metric of journals' scientific prestige: the SJR indicator // Journal of Informetrics 2010, vol.4, no.3, pp.379-391.

6. Perez-Hornero P., Arias-Nicolas J.P., Pulgarin A.A. An annual JCR impact factor calculation based on Bayesian credibility formulas // Journal of Informetrics, 2013, vol.7, no.1, pp.1-9.

7. Rousseau R. On the relation between the WoS impact factor, the Eigenfactor, the SCImago Journal Rank, the Article Influence Score and the journal h-index. Preprint 2009. http://eprints.rclis.org/13304/

8. Palacios-Huerta I., Volij O. The measurement of intellectual influence // Econometrica, 2004, vol.72, no.3, pp.963-977.

9. Bergstrom C. Eigenfactor: Measuring the value and prestige of scholarly journals // College \& Research Libraries News, 2007, vol.68, no.5, pp.314-316.

10. Bollen J., van de Sompel H., Hagberg A., Chute R. A principal component analysis of 39 scientific impact measures // PLoS ONE, 2009, vol.4, no.6, e6022: doi:10.1371/journal.pone.0006022

11. Lillquist E., Green S. The discipline dependence of citation statistics // Scientometrics, 2010, vol.84, no.3, pp.749-762.

12. Owlia P., Vasei M., Goliaei B., Nassiri I. Normalized impact factor (NIF): an adjust method for calculating the citation rate of biomedical journals // Journal of Biomedical Informatics, 2011, vol.44, no.2, pp.216-220.

13. Iftikhar M., Masood S., Song T.T. Modified impact factor (MIF) at specialty level: a way forward // Procedia - Social and Behavioral Sciences, 2012, vol.69, pp.631-640.

14. Pudovkin A., Garfield E. Rank-normalized impact factor: a way to compare journal performance across subject categories // Proceedings of the American Society for Information Science and Technology 2004, vol.41, no.1, pp.507-515. 
15. Leydesdorff L, Opthof T. Scopus' source normalized impact per paper (SNIP) versus the journal impact factor based on fractional counting of citations // Journal of the American Society for Information Science and Technology, 2010, vol.61, no.11, pp.2365-2396.

16. Pinski G., Narin F. Citation influence for journal aggregates of scientific publications: theory, with application to literature of physics // Information Processing and Management, 1976, vol.12, no.5, pp.297-312.

17. Bollen J., Rodriguez M.A., van de Sompel H. Journal status // Scientometrics, 2006, vol.69, no.3, pp.669-687.

18. Zyezkowski K. Citation graph, weighted impact factor and performance indices // Scientometrics, 2010, vol.85, no.1, pp.301-315.

19. Buela-Casal G. Assessing the quality of articles and scientific journals: proposal for weighted impact factor and a quality index // Psychology in Spain, 2004, vol.8, no.1, pp.60-76.

20. Habibzadeh F, Yadollahie M. Journal weighted impact factor: a proposal // Journal of Informetrics, 2008, vol.2, no.2, pp.164-172.

21. Waltman L., Van Eck N.J. Some comments on the journal weighted impact factor proposed by Habibzadeh and Yadollahie // Journal of Informetrics, 2008, vol.2, no.4, pp.369-372. 